\title{
Pricing Bitcoin under Double Exponential Jump-Diffusion Model with Asymmetric Jumps Stochastic Volatility
}

\author{
Ndeye Fatou Sene', Mamadou Abdoulaye Konte ${ }^{2}$, Jane Aduda ${ }^{3}$ \\ ${ }^{1}$ Department of Mathematics, Pan African University Institute of Basic Sciences, Technology and Innovation, Nairobi, Kenya \\ ${ }^{2}$ Department of Economics, L.A.R.E.S, Gaston Berger University, Saint-Louis, Senegal \\ ${ }^{3}$ Department of Statistics and Actuarial Sciences, Jomo Kenyatta University of Agriculture and Technology, Nairobi, Kenya \\ Email: ndeyefatousene1502@gmail.com
}

How to cite this paper: Sene, N.F., Konte, M.A. and Aduda, J. (2021) Pricing Bitcoin under Double Exponential Jump-Diffusion Model with Asymmetric Jumps Stochastic Volatility. Journal of Mathematical Finance, 11, 313-330.

https://doi.org/10.4236/jmf.2021.112018

Received: March 13, 2021

Accepted: May 28, 2021

Published: May 31, 2021

Copyright (๑) 2021 by author(s) and Scientific Research Publishing Inc. This work is licensed under the Creative Commons Attribution International License (CC BY 4.0).

http://creativecommons.org/licenses/by/4.0/

\begin{abstract}
The objective of this study is, to show the importance of incorporating jumps in both returns and volatility dynamics for Bitcoin. For that purpose, we introduce the Double Exponential Jump-Diffusion model with Stochastic Volatility (DEJDSVJ) that contains asymmetric jumps. The use of the Markov Chain Monte Carlo methods for estimation has proved the meaningful presence of jumps in Bitcoin price and volatility. Moreover, based on the Bitcoin options market, a comparison between the underlying model, the Double Exponential Jump Diffusion model (DEJD) with Stochastic Volatility (no Jumps) and the Stochastic Volatility (SV) shows the goodness of the DEJDSVJ model's calibration over others for pricing Bitcoin options.
\end{abstract}

\section{Keywords}

Bitcoin, Double Exponential Jump-Diffusion, Stochastic Volatility, Markov Chain Monte Carlo

\section{Introduction}

Bitcoin is a digital currency that satisfies the technical properties of money. Contrary to fiat currencies, there is no central authority acting as a bank for Bitcoin. Its system is based on solving computational algorithms (cryptographic puzzles) known as mining process through a network called blockchain whose protocol was released by a pseudonymous Satoshi Nakamoto on 2009 [1]. Since it was generated, the price of bitcoin in USD dollar varies over time. This last decade, the fluctuations of Bitcoin's price raised a lot of attention for investors. 
Many questions are asked about the future of bitcoin. For some people, bitcoin does not fill all aspects of money that are medium of exchange, store value and a unit of account. Critical mass supports that Bitcoin is not a universal medium of exchange as it has small user base. Also, some economists see it as a risky investment instead of a stable store of value because it is volatile.

But despite those critics, some studies proved that there will be a future role of Bitcoin in financial markets. [2] studied the contributions of Bitcoin exchange on price discovery, [3] analyzed the GARCH volatility of bitcoin. Also [4] showed that bitcoin can be used as a speculative asset instead of medium of exchange. In addition, on December 18, 2017, the Chicago Mercantile Exchange (CME) and Chicago Board Options Exchange (CBOE) launched futures for Bitcoin. Also the US regulator considers it as a commodity. So, it becomes interesting to do study about pricing and hedging Bitcoin derivatives. Doing so will help investors to come with efficient risk management and portfolios selection strategies.

Up to now, few academic researches were done in this direction because of some challenges that concern the characteristics of Bitcoin: price discontinuity, high level of speculation, high volatility.

Figure 1 shows the discontinuities in price of Bitcoin. The graph reveals extreme price variations on 2017 and many fluctuations.

To fulfill such challenges, it is important to model the dynamic of BTC including the occurrence of rare or extreme events as jump process in returns. In literature about options pricing model, many studies were done using diffusion

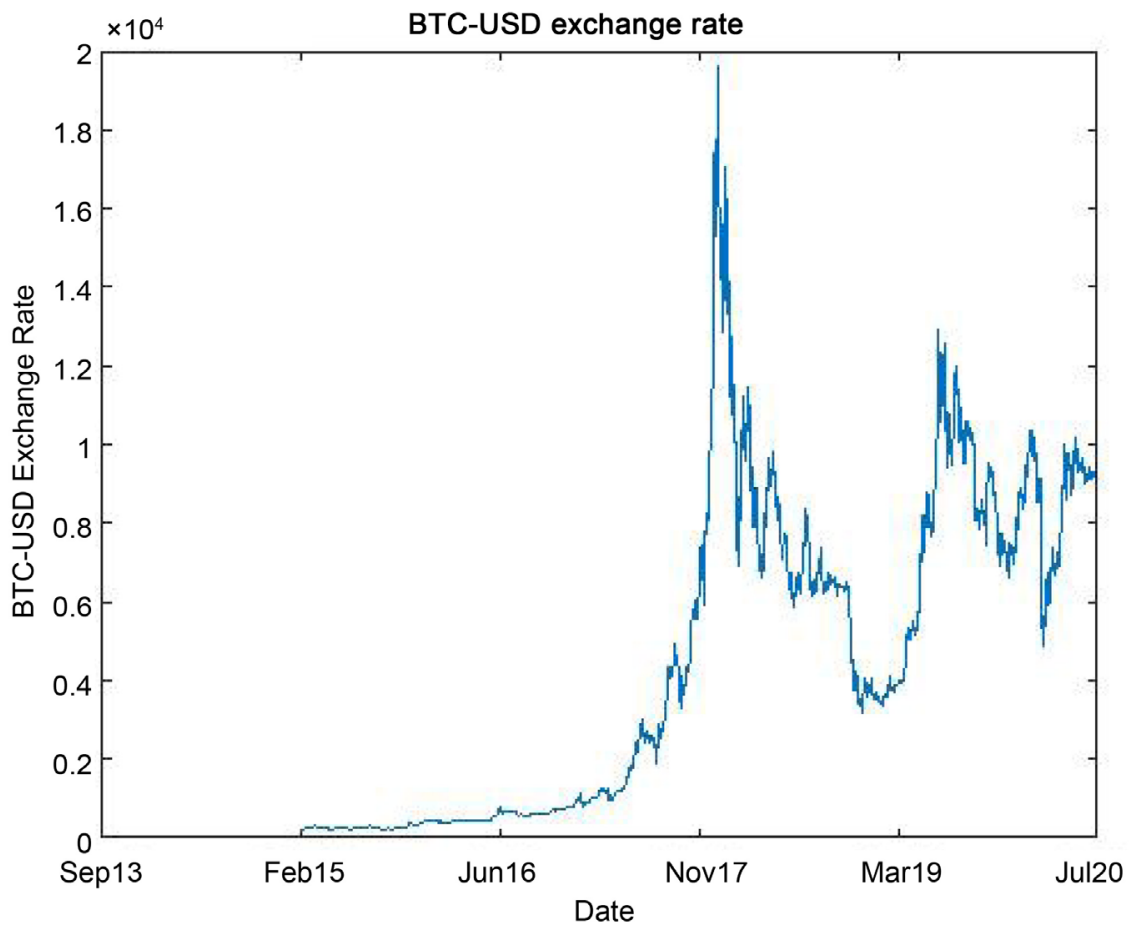

Figure 1. USD-BTC exchange rate from Coin base market over the period of February 2015 to July 2020. 
models with the geometric Brownian motion process [5]. However, such models do not incorporate all facts that intervene on data. Thus, some authors have chosen to introduce the jump-diffusion models in which the jumps capture all facts [6] [7].

In addition, the characteristic of the volatility is important for option pricing models. Volatility plays an important role in pricing of derivatives. After the financial crisis on 1987, many studies have demonstrated that the inefficiency of the Black-Scholes model [5] is caused by the constant volatility. Since that, studies came with many models of volatility in which the variance is randomly distributed such as the stochastic volatility [8]. [8] considered European call options price with stochastic volatility and also stochastic interest rates. He found the importance of correlation between price and volatility in order to explain the return skewness and strike price biases in Black-Scholes model. So, stochastic volatility models are considered as models that match well the fair value of an option.

Although few studies are done about bitcoin options pricing, we find many relevant considerations in some articles. [9] proposed an option pricing model under double exponential jump diffusion with mean reverting stochastic volatility and stochastic interest rate. The model considered the leptokurtosis and heteroscedasticity. They checked the goodness of fit of the model using the 50 ETF data exhibited from the Shanghai Stock Exchange and compared the model with both [6] and [5] models. The results gave a high pricing accuracy in such model rather than the other models. [10] tested the goodness of the model of [6] using Bitcoin as the underlying asset. The outcomes gave a good fit of market prices by the model in [6] but comparing it with the Black-Scholes model [5]; he found that there is not much difference between the results. [7] proposed a model under the stochastic volatility with correlated jumps (SVCJ) on both returns and volatility. They found the importance of taking into account the jumps for the cryptocurrency derivatives markets. Furthermore, with the development of Artificial Intelligence, [11] introduced an option model based on non parametric method developed on neural network and he calibrated it with the parametric methods such as Tree Trinomial, Finite Difference and Monte Carlo simulation.

In this article, we contribute to the growing academic literature about Bitcoin derivative markets by considering the jump diffusion model of [6]. [6] introduced a jump-diffusion model for option pricing in which the jump's sizes are double exponentially distributed. The benefits when using such distribution in jump is to capture the asymmetric leptokurtic effect and volatility smile. Also [6] were able to find an analytical option price formula of the model contrary to the models where the jump's sizes are not double exponentially distributed.

Our difference with [6] model is that, we add asymmetric jumps into the Stochastic Volatility process. Thus, the aim of this paper is to study the Double Exponential Jump Diffusion model with Stochastic Volatility that contains Jumps (DEJDSVJ). Another difference with [7] is the distribution of the jumps as we consider the double exponential distribution and also, the jump are not corre- 
lated.

The paper is presented as follows. In Section 2, we analyze the dynamic of Bitcoin and introduce the model. In Section 3, we estimate the parameters and interpreted the results. We implement in Section 4 the option pricing exercises and compare the Double Exponential Jump-Diffusion with asymtric jump Stochastic Volatility (DEJDSVJ) model with the the Double exponential Jump Diffusion model with Mean Reverting Stochastic Volatility (DEJD) (i.e. no jumps in volatility) and the Stochastic Volatility (SV) model meaning the absence of jumps in both returns and volatility processes and finally, we graph and analyze the Implied Volatility surface in Section 5.

\section{Dynamic of Bitcoin}

Currently, Bitcoin knows a huge emergence. Traders and investors give it more attention because it is not controlled by a central authority. The technology behind Bitcoin is an open source. Its properties are transparency, anonymous, fast transactions and cheap. Also, there is not prerequisites or interest rate for governments or banks. All transactions made are registered by the blockchain system whose functionnement depends on mining process. Since it was established, the price in USD dollar (exchange rate) of Bitcoin fluctuates by going either up or down. Thus, it generates a fear sentiment for some people to invest on it. Some studies have tried to investigate about the factors that drive the price ([12] [13]). The most common macroeconomic variables that were found are the supply of bitcoin, the media attention, interest rates.

\subsection{Analysis of Bitcoin Price, Returns and Volatility}

To analyze such dynamic, we use a daily time series data exhibited from the database of COINBASE exchange platform one of the popular largest market of bitcoin based at USA. The data cover the time period from February 2015 to July 2020. We use a big sample of data in order to avoid bias. Figure 1 depicts the bitcoin price chart with scattered spikes.

It directly shows that the time series of the underlying is not stationary; there are discontinuities. The graph depicts many phases:

- From February 2015 to April 2017, the graph fit to a very flat curve line; that period is considered as normal because there is not high fluctuations of the BTC-USD values.

- From May 2017 to November 2017, data exhibit some steeply fluctuations. Regular ups and downs spikes are observed.

- However the end of 2017 is marked by unregular increasing spike of the exchange rate that reaches for the first time a value around $\$ 20,000$. Such change can be defined as an irregular component because it was unpredictable. During that period, the interest of media and governments about cryptocurrencies increased.

- After that, we notice a mildly decreasing curve up to half of 2019 again fol- 
lowed by some fluctuations of BTC-USD exchange rate.

The aspect of the Geometric Brownian Motion (GBM) process is directly observed. It represents the continuity part of a process. Generally, GBM assumes that a constant drift is accompanied by random shocks.

Many options pricing models consider a given underlying asset as a continuous process (Black Scholes model [5], Heston model [8], etc.). However, empirical analysis of data showed the presence of discontinuity on many processes like stock price, exchange rate, etc. Those discontinuities are defined as jumps processes. Jump processes provide more realistic results. The importance of jump-diffusion model is its ability to capture stylized facts as skew, leptokurtosis, volatility smile/skew etc as shown by [6]. Furthermore, many studies have shown the presence of jumps on bitcoin dynamics ([14] [15]) and therefore they are incorporated on pricing Bitcoin derivatives.

As the price is nonstationary, we take the log returns as a response variable. Log returns are the first difference of the logarithm of the prices (see Equation (1)).

$$
r_{t}=\ln \left(P_{t}\right)-\ln \left(P_{t-1}\right)
$$

where:

$r_{t}$ is the log returns at time $t$.

$P_{t}$ is the price of Bitcoin in USD at time t. Here, we took the log returns as percentage meaning that we multiply $r_{t}$ by 100. In Figure 2, we have plotted the daily log returns of Bitcoin for the same data sample.

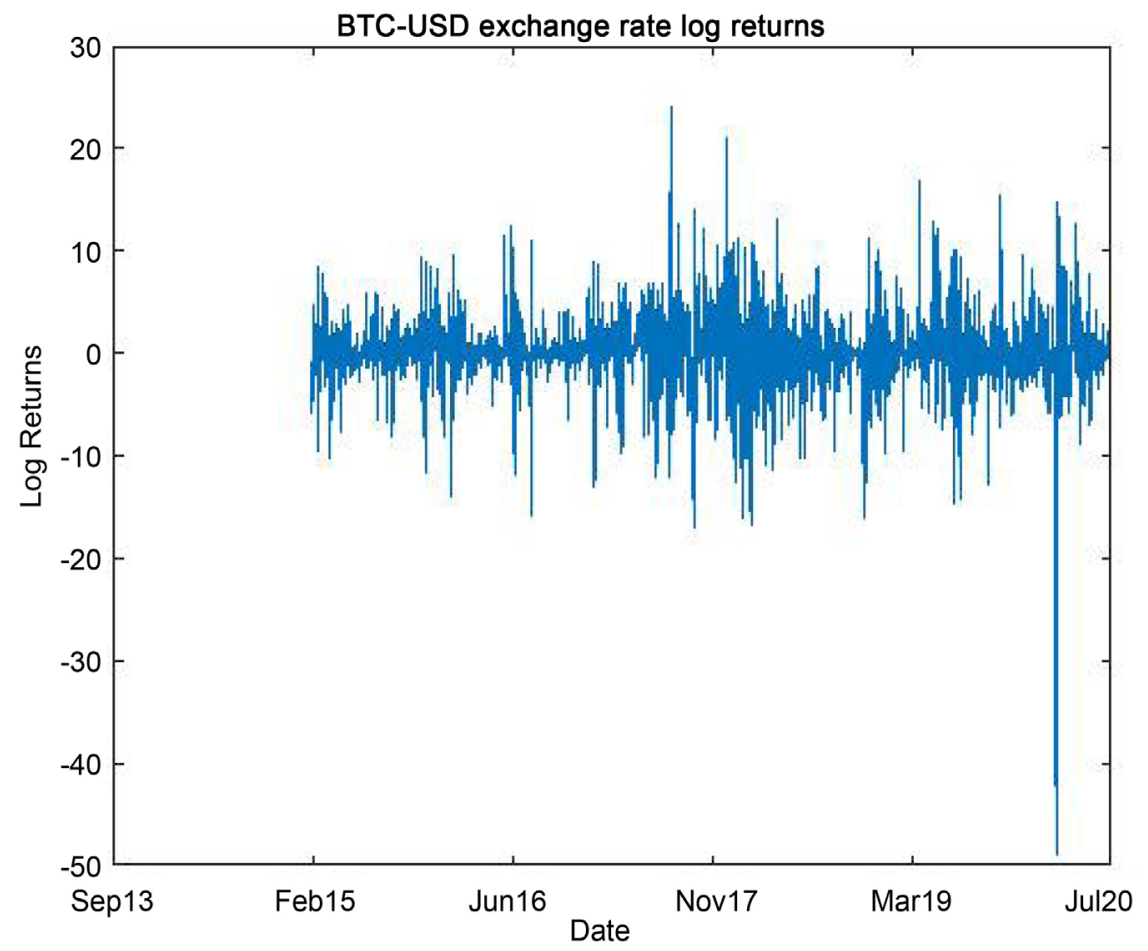

Figure 2. Daily log returns (percent \%) of Bitcoin from Coin base market over the period of February 2015 to July 2020. 
The series of returns fluctuates around zero. Figure 2 depicts sustained periods of low or high volatility; it shows an effect of volatility clustering meanig that small returns are followed by small returns and large returns are followed by large returns. From 2015 to 2017, the values of returns are almost the same and slow. By 2017, we have high values. That sudden change in returns can be explained by the fact that the price was too high by that year.

Thus, the residual is conditionally heteroskedastic. The use of stochastic volatility in our work is supported by such effect of clusters. Also, [16] proved that the local and the time dependent volatility model do not necessarily capture the volatility clusters. Furthermore, the results of [17] showed that the dynamic of bitcoin is better modeled by the stochastic volatility model than the GARCH volatility.

In Figure 3, we depict the volatility of the same data frame used for the log returns. An analysis of such data gives an evidence that the volatility tends to fluctuate. Graphically, from the beginning to year 2017, we observe standard fluctuations (up and down movements). By end of 2017, there is a high move of the volatility that reverts down by 2018 .

\subsection{The Model}

Here, we introduce the jump diffusion process with Stochastic Volatility Jump to

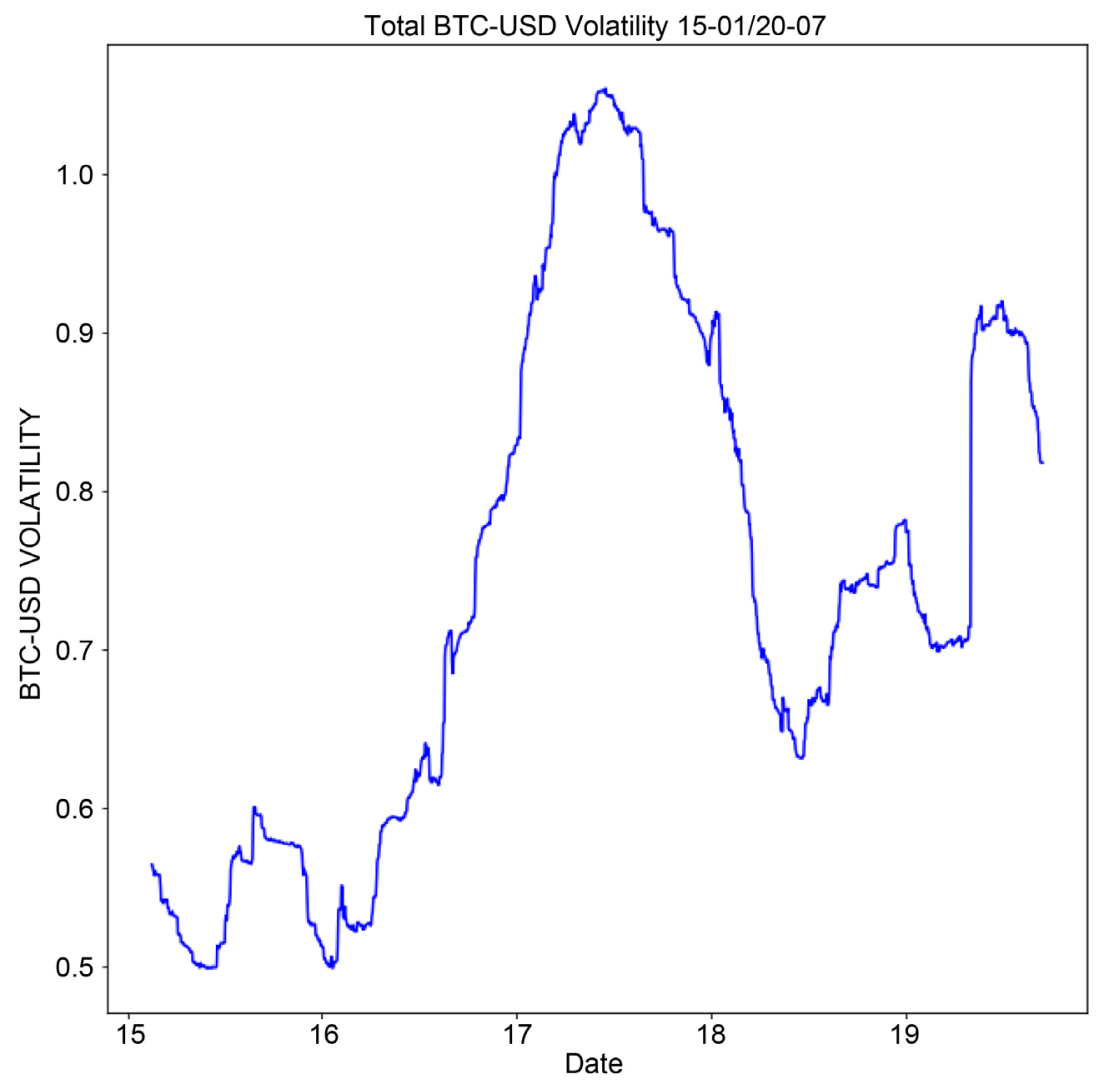

Figure 3. Bitcoin volatility from coinbase market over the period of February 2015 to July 2020. 
model the dynamic of bitcoin.

Let define $S_{t}=\mathrm{e}^{X_{t}}$ with $S$ the underling process of BTC-USD price and $X$ is the basic state process such that:

$$
\mathrm{d} X_{t}=\mu \mathrm{d} t+\sqrt{V_{t}} \mathrm{~d} W_{t}^{1}+\left(\mathrm{e}^{y_{t}^{1}}-1\right) \mathrm{d} N_{t} .
$$

where $\mu$ is the constant drift term, $V$ is the Stochastic Volatility with Jump defined as:

$$
\mathrm{d} V_{t}=\kappa\left(\phi-V_{t}\right) \mathrm{d} t+\sigma \sqrt{V_{t}} \mathrm{~d} W_{t}^{2}+\left(\mathrm{e}^{y_{t}^{2}}-1\right) \mathrm{d} N_{t} .
$$

$\mathrm{d} W^{1}, \mathrm{~d} W^{2}$ are the Brownian motion processes that are correlated. $y_{t}^{1}, y_{t}^{2}$ are independent double exponentially distributed random variables such that:

$$
\begin{aligned}
& f\left(y^{1}\right)=p \eta_{1} \mathrm{e}^{-\eta_{1} y^{1}} I_{\left\{y^{1} \geq 0\right\}}+(1-p) \eta_{2} \mathrm{e}^{-\eta_{2} y^{1}} I_{\left\{y^{1}<0\right\}} \\
& f\left(y^{2}\right)=q \eta_{1}^{\prime} \mathrm{e}^{-\eta_{1}^{\prime} y^{2}} I_{\left\{y^{2} \geq 0\right\}}+(1-q) \eta_{2}^{\prime} \mathrm{e}^{-\eta_{2}^{\prime} y^{2}} I_{\left\{y^{2}<0\right\}}
\end{aligned}
$$

where $\eta_{1}>1, \eta_{2}>0, \eta_{1}^{\prime}>0$ and $\eta_{2}^{\prime}>0$.

$\mathrm{d} N$ is a pure jump process following a poisson distribution with constant mean jump arrival rate $\lambda$.

Let $(\Omega, \mathbb{F}, \mathbb{P})$ be the filtered probability space. Then using Itô's formula under the probability measure $\mathbb{Q}$, we introduce the diffusive price process $d S_{t}$ of [6]

$$
\begin{aligned}
\log \left(S_{t}\right) & =\log \left(\mathrm{e}^{X_{t}}\right) \\
\mathrm{d}\left(\log \left(S_{t}\right)\right) & =\mathrm{d}\left(X_{t}\right) \\
\mathrm{d} S_{t} & =S_{t-}\left(\mu \mathrm{d} t+V_{t} \mathrm{~d} W_{t}^{1}+\left(\mathrm{e}^{y_{t}^{1}}-1\right) \mathrm{d} N_{t}\right)
\end{aligned}
$$

By combining the above process with the stochastic volatility, we obtain the following dynamic model:

$$
\left\{\begin{array}{l}
\frac{\mathrm{d} S_{t}}{S_{t-}}=\mu \mathrm{d} t+\sqrt{V_{t}} \mathrm{~d} W_{t}^{1}+Z_{t}^{1} \mathrm{~d} N_{t} \\
\mathrm{~d} V_{t}=\kappa\left(\phi-V_{t}\right) \mathrm{d} t+\sigma \sqrt{V_{t}} \mathrm{~d} W_{t}^{2}+Z_{t}^{2} \mathrm{~d} N_{t} \\
\operatorname{cov}\left(\mathrm{d} W_{t}^{1}, \mathrm{~d} W_{t}^{2}\right)=\rho \mathrm{d} t \\
P\left(\mathrm{~d} N_{t}=1\right)=\lambda \mathrm{d} t \\
Z_{t}^{1}=Y_{t}^{1}-1 \\
Y_{t}^{1}=\mathrm{e}^{y_{t}^{1}} \\
Z_{t}^{2}=Y_{t}^{2}-1 \\
Y_{t}^{2}=\mathrm{e}^{y_{t}^{2}}
\end{array}\right.
$$

$S$ is the exchange rate (BTC-USD price), $V$ is the stochastic variance with asymmetric jumps. $\kappa$ is the mean reversion rate or the degree of volatility clustering, $\phi$ is the long-run mean of $V$; the process reverts to that level with a spread governed by $\kappa$.

$W_{t}^{1}$ and $W_{t}^{2}$ are two Brownian motion $\rho$-correlated; they represent respec- 
tively the diffusion processes for the $S$ and $V$,

$N$ is a pure jump process following a poisson distribution with constant mean jump arrival rate $\lambda$ (i.e. $\left.\mathbb{P}_{k}(\lambda \mathrm{d} t)=\mathbb{P}\left(\mathrm{d} N_{t}=k\right)=\frac{\mathrm{e}^{-\lambda \mathrm{d} t}(\lambda \mathrm{d} t)^{k}}{k !}\right)$.

$\sigma$ is the volatility of volatility that controls the kurtosis.

$\rho$ the correlation coefficient between the log returns and the volatility that affects the asymmetry or skewness. As there is not dividend yields on Bitcoin, we will not consider such parameter in the drift term of the log return process.

Under the square root variance process, the model allows for systematic volatility risk.

\section{Markov Chain Monte Carlo Estimation}

\subsection{Procedure}

For the above model to be applicable in order to get option prices in following section, we have to estimate the values of parameters and the latent variables. Since [6] has introduced the double exponential jump diffusion model, many methods for estimation of the model's parameters were used. Among them, we have the Maximum Likelihood Estimation (MLE) method ([18]), the Generalized Method of Moment (GMM) ([19]), the Empirical Characteristic Exponent (ECE) ([20]), the Markov Chain Monte Carlo(MCMC) method ([9]) etc. In this study, we also consider the MCMC method. Our motivation is its efficiency to identify the latent variables such that the stochastic volatility, the jump sizes and the jump. First used in stochastic volatility model's estimation by [21], MCMC method was extended in jump-diffusion models by [22] and it becomes very popular in quantitative finance.

Markov Chain Monte Carlo methods are a class of algorithms that allows to find a posterior distribution and to sample from it a Markov chain for the set of parameters. Primarly, such methods were used in Bayesian statistics, computational physics, computational linguistics in order to address multi-dimensional problems.

MCMC becomes popular in field of quantitative finance this year because of its ability to estimate the parameters of complex dynamic models that are developed to solve the problems in finance such as risk management and options pricing. The most meaningful part of the MCMC methods is the approximation of the posterior. Also called the conditional joint distribution of random variables (parameters and latent variables) given the data, the posterior is derived from Baye's formula such that:

$$
P(\theta, L \mid Y) \propto P(Y \mid \theta, L) P(\theta, L) .
$$

where $Y$ is the data, $\theta$ is the set of parameters of a given model and $L$ represents the latent variables.

After setting the initial values and the prior for each parameter, there are two approaches to get the posterior for each parameter: the Gibbs sampling method 
and the Metropolis-Hastings M-H algorithm. For the former, it is used when the posterior distribution derived can be approximated to a known probability distribution or in case of conjugate posterior that means the prior and the posterior for a given parameter have the same distributions with different input hyperparameters. The $\mathrm{M}-\mathrm{H}$ algorithms is considered when there is not a standard distribution that links with the posterior. A good overview of the MCMC methods can be found in [23].

In what follows, we highlight the different steps to consider before implementing the MCMC algorithm in Matlab software.

First of all, we discretize both the log returns and the stochastic volatility under Euler-Maruyama discretization method.

$$
\begin{aligned}
\Delta S_{t}= & S_{t-1}\left(\mu \Delta t+\sqrt{V_{t}} W^{1}(\Delta t)+Z_{t}^{1} J_{t}\right) \\
\Rightarrow & r_{t}=\mu \Delta t+\sqrt{V_{t}} B_{t}^{1}+Z_{t}^{1} J_{t} \\
& r_{t}=\frac{\Delta S_{t}}{S_{t-1}} . \\
\Delta V_{t}= & V_{t}-V_{t-\Delta t} \\
\Rightarrow V_{t}= & \left(\alpha+\beta V_{t-1}\right) \Delta t+\sigma \sqrt{V_{t-1}} W^{2}(\Delta t)+Z_{t}^{2} J_{t} \\
\Rightarrow V_{t}= & \left(\alpha+\beta V_{t-1}\right) \Delta t+\sigma \sqrt{V_{t-1}} B_{t}^{2}+Z_{t}^{2} J_{t}
\end{aligned}
$$

where $t \in 1,2, \cdots, T$ is discrete daily time $(\Delta t=1), \alpha=\kappa \phi$ and $\beta=1-\kappa$, $P\left(J_{t}=1\right)=\lambda \Delta t$.

$B^{1}$ and $B^{2}$ are respectively the standardized residuals of the diffusive processes $W^{1}$ and $W^{2}$. Regarding the properties of a geometric brownian motion, $W(t)$ follows a gaussian distribution $\mathbb{N}(0, t)$. Hence, it has the same distribution than $\sqrt{t} B^{i}$ with $B_{i} \sim \mathbb{N}(0,1), i \in\{1,2\}$.

The discretized model becomes:

$$
\left\{\begin{array}{l}
r_{t}=\mu+\sqrt{V_{t}} B_{t}^{1}+Z_{t}^{1} J_{t} \\
V_{t}=\alpha+\beta V_{t-1}+\sigma \sqrt{V_{t-1}} B_{t}^{2}+Z_{t}^{2} J_{t}
\end{array}\right.
$$

After the discretization, we assume $\theta_{1}=\left\{\mu, \lambda, \eta_{1}, \eta_{2}, p\right\}$ the set of parameters in returns, $\theta_{2}=\left\{\alpha, \beta, \sigma, \eta_{1}^{\prime}, \eta_{2}^{\prime}, q\right\}$ contains the parameters of the stochastic volatility and $\Sigma=\left\{V, Z^{1}, Z^{2}, J\right\}$ the set for the latent variables.

Thus, $\theta=\theta_{1} \cup \theta_{2} \cup\{\rho\}=\left\{\mu, \lambda, \eta_{1}, \eta_{2}, \eta_{1}^{\prime}, \eta_{2}^{\prime}, p, q, \alpha, \beta, \sigma, \rho\right\}$ represents the set of all parameters to estimate.

Thus, we define the posterior distribution based on Baye's formula to respect the principle of MCMC method. We obtain:

$$
\begin{gathered}
P\left(\theta, V, Z^{1}, Z^{2}, J \mid r\right)=P\left(r \mid \theta, V, Z^{1}, Z^{2}, J\right) \times P\left(\theta, V, Z^{1}, Z^{2}, J\right) \\
P\left(\theta, V, Z^{1}, Z^{2}, J \mid r\right)=P\left(r \mid \theta, V, Z^{1}, Z^{2}, J\right) \times P(\theta) \times P\left(V, Z^{1}, Z^{2}, J \mid \theta\right)
\end{gathered}
$$

$P\left(r \mid \theta, V, J, Z^{1}, Z^{2}\right)$ represents the likelihood of the data,

$P\left(V, J, Z^{1}, Z^{2} \mid \theta\right)$ is the prior of the latent variables given their parameters respectively. 
$P(\theta)$ is the prior of the parameters.

Furthermore, we assume that all parameters have mutually independent prior distributions. That implies

$$
P(\theta)=P\left(\theta_{1}\right) P\left(\theta_{2}\right) P(\rho)
$$

where

$$
\begin{gathered}
P\left(\theta_{1}\right)=P(\mu) P\left(\eta_{1}\right) P\left(\eta_{2}\right) P(\lambda) P(p) \\
P\left(\theta_{2}\right)=P(\alpha) P(\beta) P(\sigma) P\left(\eta_{1}^{\prime}\right) P\left(\eta_{2}^{\prime}\right) P(q)
\end{gathered}
$$

Based on relevant studies [7] [9] [24], we consider the following prior distributions: $\mu \sim N(0,25), \lambda \sim \operatorname{Beta}(2,40), \eta_{1} \sim I W(10,40), \quad \eta_{2} \sim I W(10,40)$, $p \sim \operatorname{Beta}(2,40), \quad(\alpha, \beta) \sim N\left(0_{2 \times 1}, I_{2 \times 2}\right), \quad \sigma \sim \ln N(2.5,0.1), \eta_{1}^{\prime} \sim \operatorname{IW}(10,40)$, $\eta_{2}^{\prime} \sim I W(10,40), \quad q \sim \operatorname{Beta}(2,40), \quad \rho \sim U(-1,1) . \quad I W$ represents the Inverted-Wishart distribution.

For the hyperparameters, we assume those in [24] and for the double exponential distribution, parameters were set under convenience to obtain the convergence of the chains.

Further, we use conjugate posterior for all parameters except $\rho$ and $\sigma$ for which we consider the $\mathrm{M}-\mathrm{H}$ algorithm. For the latent variables, the posteriors of the jump sizes $Z^{1}$ and $Z^{2}$ follow a double exponential distribution. The jump $J$ has a Bernouilli distribution and the volatility $V$ has a non stantard posterior; so we use the $\mathrm{M}-\mathrm{H}$ algorithm to approximate it.

\subsection{Numerical Results and Analysis}

To estimate the above parameters under MCMC method, we consider a set of 2000 daily data points from the Coinbase market. We use large sample of data to avoid bias. We run $N=5000$ iterations with a burn-in of $n=500$ iterations. We have chosen such burn-in period to remove the effects of initial values that affect only five hundred instead of one of the different chains. The value of each parameter $x$ is obtained by averaging the sum of the posterior's values over $N-n$ iterations:

$$
x=\frac{1}{N-n} \sum_{i=n+1}^{N} x_{i}
$$

Hence, Table 1 contains the estimated value of the underlying model and two others models that are later used for comparison.

We have the leverage effect with a negative value of $\rho$. It means that bad news about the market of BTC increase the price of such asset. Thus, we can explain the huge fluctuations of the price this last year by the attention about Bitcoin. Although, some investors defend the importance of using Bitcoin, there are a lot of critics about it supported by governments and banks that do not trust the digital currency. The values $\eta_{1}$ and $\eta_{2}$ show the interest of incorporating the jumps in returns. In addition, the values of $\alpha$ and $\beta$ exhibit an important value of spread $\kappa$ and mean $\phi$ of revesion. So, the Stochastic Volatility is very useful for BTC price. 
Table 1. MCMC parameter's value of the three models using BTC daily return from January 29, 2015 to July 19, 2020.

\begin{tabular}{cccc}
\hline Parameters & DEJD & DEJDSVJ & SV \\
\hline$\mu$ & 0.1839 & 0.1542 & 0.1843 \\
$\eta_{1}$ & 52.9287 & 49.2762 & - \\
$\eta_{2}$ & 42.0980 & 33.0683 & - \\
$\eta_{1}^{\prime}$ & - & 43.9765 & - \\
$\eta_{2}^{\prime}$ & - & 39.6936 & - \\
$\lambda$ & 0.0011 & 0.0028 & - \\
$\alpha$ & 12.1648 & 12.0071 & 12.1641 \\
$\beta$ & -0.7824 & -0.7736 & -0.7825 \\
$\rho$ & -0.0871 & -0.1084 & -0.0878 \\
$\sigma$ & 0.3532 & 0.4 & 0.353 \\
$p$ & 0.0009 & 0.0025 & - \\
$q$ & - & 0.0024 & - \\
\hline
\end{tabular}

After estimation, we checked the sensitivity of the posterior regarding the prior distributions assumption. It concerns the convergence of each chain. For that purpose, we graph the trace plot of each chain with respect to the simulation index.

Figure 4 contains the twelve trace plots that represent each chain for each parameter of the DEJDSVJ model. Each graph proves the convergence for one of the parameters.

We obtain quick convergence for each parameter (after 500 iterations over 5000). Thus, the prior distributions and the likelihood function of the data given the parameters are well chosen. The data too are well sampled.

In the following section, the parameters values of the models are used to price European call options.

\section{Mechanism of European Call Option Pricing}

\subsection{Monte Carlo Simulations of the DEJDSVJ Model}

An option pricing model involves a probabilistic approach to assign a fair value for an option. An option is a contract that gives the holder the right but not the obligation to buy (call option) or to sell (put option) an underlying security at a pre-determined price at (European option) or before (American option) a maturity time. The variables considered to price options are the current market price, the strike price, the volatility, the interest rate and time to maturity.

Let $\mathbb{Q}$ be the probabilty measure under which options are priced.

Let $S(t)$ be the price a time $t, r$ the interest rate, $K$ the strike and $T$ the expiry time.

The European Call Option price is defined as follow:

$$
C(t)=\mathbb{E}^{\mathbb{Q}}\left(\mathrm{e}^{-r(T-t)}(S(T)-K)^{+}\right)
$$




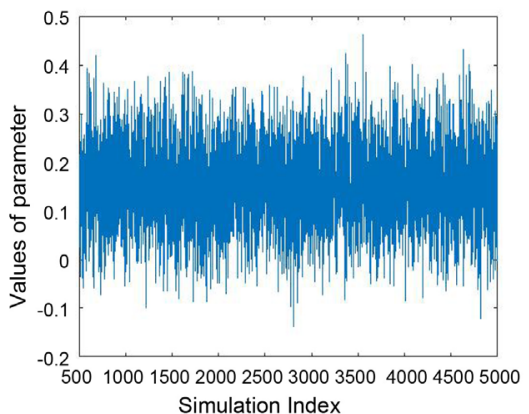

(a) $\mu$

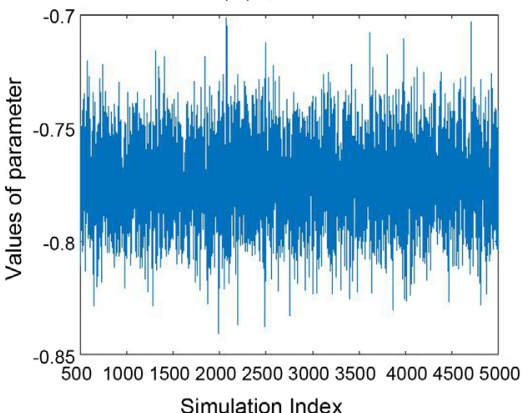

(d) $\beta$

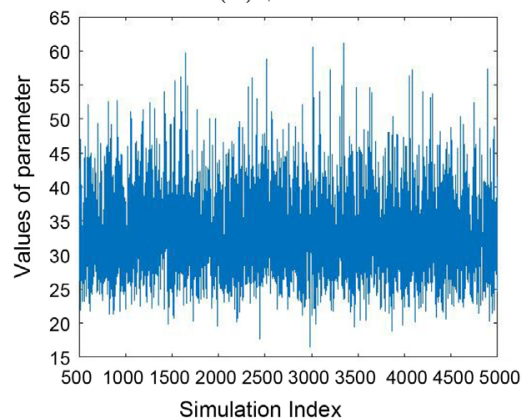

(g) $\eta_{2}$

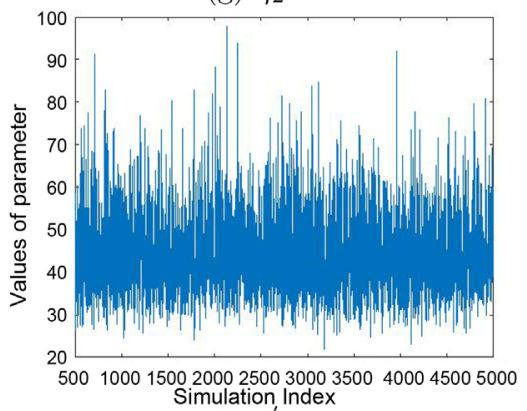

(j) $\eta_{1}^{\prime}$

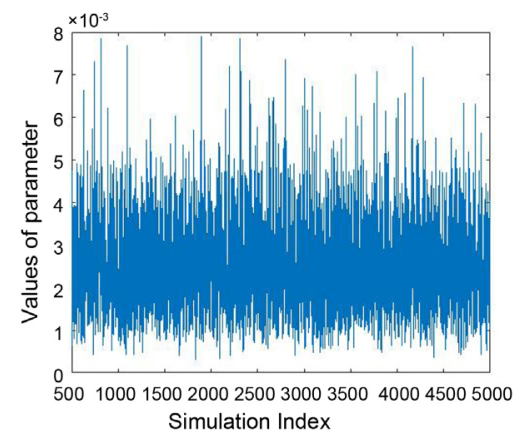

(b) $\lambda$

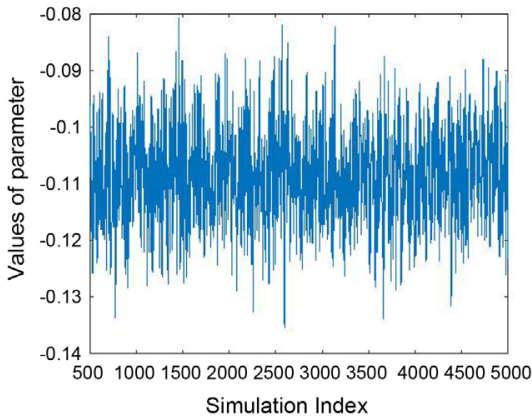

(e) $\rho$

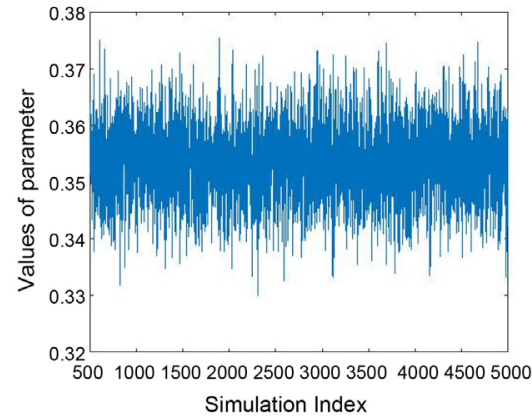

(h) $\sigma$

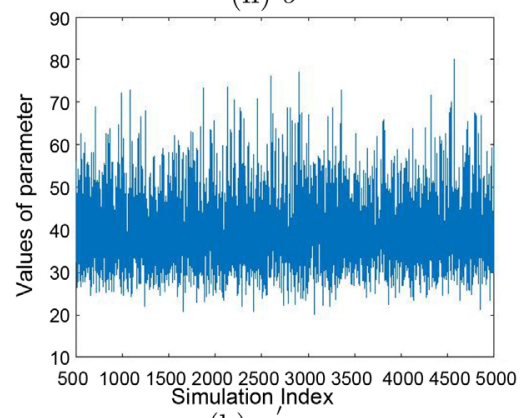

(k) $\eta_{2}^{\prime}$

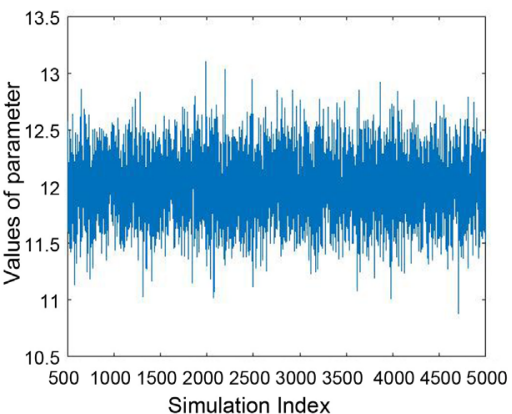

(c) $\alpha$

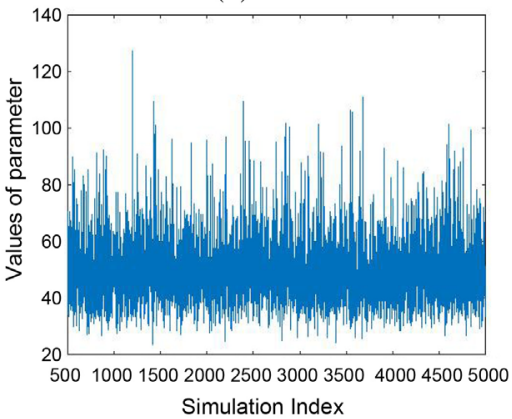

(f) $\eta_{1}$

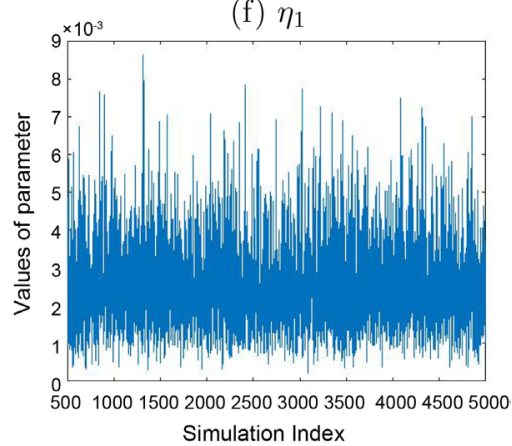

(i) $p$

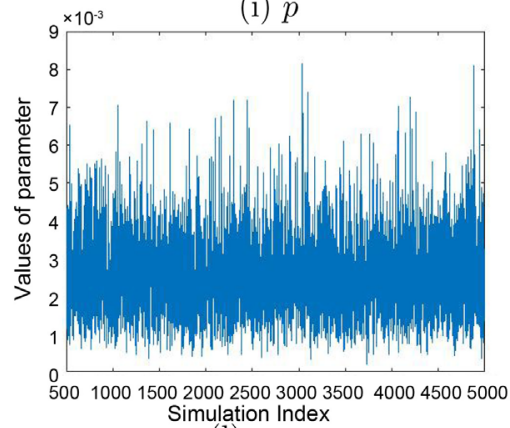

(l) $q$

Figure 4. MCMC trace plots of the parameter samples for the DEJDSVJ model. (a) $\mu$, (b) $\lambda$, (c) $\alpha$, (d) $\beta$, (e) $\rho$, (f) $\eta_{1}$, (g) $\eta_{2}$, (h) $\sigma$, (i) $p$, (j) $\eta_{1}^{\prime}$, (k) $\eta_{2}^{\prime}$, (l) $q$.

where, $(S(T)-K)^{+}=\max (S(T)-K, 0)$ represents the payoff $\phi(T)$ of the option at $T$. For the call option to have a value, $S(T)$ should be greater than $K$; otherwise, it is called a worthless Call Option. Many models are developed to value options such as the benchmark Black-Scholes Merton model [5], the Binomial option pricing, the Monte Carlo simulation etc. We are motivated by the 
later due to its efficiency to simulate price paths for high dimensional stochastic processes that have sources of uncertainty. The M.C simulation is based on the Euler-Maruyama discretization of the dynamic model. Knowing the price at $t=0$, the Strike $K$, the interest rate $r$ and $T$ the maturity in term of years, we compute the steps below to get an approximation of the European Call Option using the parameters in Table 1 for the model:

- We choose two large numbers $M$ the number of M.C simulations for price and $N$ the number of time steps.

- $\Delta t=\frac{T}{N}$ is the time steps.

For $i=1, \cdots, M$ :

- We generate the sources of uncertainty (randomness) $B_{i}^{1}$ and $B_{i}^{2}$ that are the standardized residuals of the discrete model. $B^{1}$ and $B^{2}$ are $\rho$-correlated. Then using the Cholesky decompositon, we construct two functions $B^{1}$ and $B^{2}$ with two independent standard normal random variables $\varepsilon^{1}$ and $\varepsilon^{2}$ such that:

$$
\left\{\begin{array}{l}
B_{i}^{1}=\varepsilon_{i}^{1} \\
B_{i}^{2}=\rho \varepsilon_{i}^{1}+\sqrt{1-\rho^{2}} \varepsilon_{i}^{2}
\end{array}\right.
$$

- We simulate the stochastic volatility $V_{i}$, the $\log$ of the price $X_{i}$ and the price $S_{i}$

$$
\begin{gathered}
V_{i}=\left(\alpha+\beta V_{i-1}\right) \Delta t+\sigma \sqrt{V_{i-1}}\left(\rho \varepsilon_{i}^{1}+\sqrt{1-\rho^{2}} \varepsilon_{i}^{2}\right) \sqrt{\Delta t}+Z_{i}^{2} J_{i} \\
X_{i}=X_{i-1}+\mu \Delta t+\sqrt{V_{i}} \sqrt{\Delta t} \varepsilon_{i}^{1}+Z_{i}^{1} J_{i} \\
S_{i}=\exp \left(X_{i}\right)
\end{gathered}
$$

- We calculate the payoff $\phi_{i}=\left(S_{i}-K\right)^{+}$and we compute the expected payoff as follow:

$$
\mathbb{E}(\phi)=\sum_{i=1}^{M} \frac{\phi_{i}}{M}
$$

- Finally, we obtain the Call Price by discounting the expected payoff:

$$
C(T)=\mathrm{e}^{-r T} \mathbb{E}(\phi)
$$

In what following, we consider the Monte Carlo Simulation described above to obtain the European Call Option Prices for the three models in order to compare them.

\subsection{Calibration}

Calibration is very important because it shows the impact of the model over the prices. To calibrate the model's call prices with the Bitcoin Options market, we consider the European call prices from the Deribit Options market on February 22, 2021 for 18 days, 32 days and 65 days to maturity. In Table 2, we made a comparison between those market's prices, the DEJDSVJ model European Call 
Table 2. Call option prices comparison.

\begin{tabular}{ccccccc}
\hline $\begin{array}{c}\text { Expiry } \\
\text { Time }\end{array}$ & $\begin{array}{c}\text { Stock } \\
\text { Prices }\end{array}$ & Strikes & $\begin{array}{c}\text { Market } \\
\text { Calls }\end{array}$ & $\begin{array}{c}\text { DEJD } \\
\text { Calls }\end{array}$ & $\begin{array}{c}\text { DEJDSVJ } \\
\text { Calls }\end{array}$ & SV Calls \\
\hline 18 days & $56,901.94$ & 54,000 & 6629.46 & 6903.9 & 6798.8 & 5835.5 \\
18 days & $56,901.94$ & 56,000 & 5605.1 & 5689.7 & 5633 & 4753.4 \\
18 days & $56,907.94$ & 58,000 & 4723.36 & 4651.1 & 4555.3 & 3826 \\
18 days & $56,907.94$ & 60,000 & 3955.47 & 3600.6 & 3664.1 & 3039.5 \\
18 days & $56,907.94$ & 62,000 & 3272.22 & 3023 & 2901.2 & 2386.1 \\
32 days & $57,716.26$ & 48,000 & $11,606.25$ & 13,259 & 12,767 & 10,680 \\
32 days & $57,716.26$ & 52,000 & 9754.54 & 10,191 & 10,074 & 7671.1 \\
32 days & $57,716.26$ & 56,000 & 7676.18 & 7577.6 & 7485.9 & 5236.4 \\
32 days & $57,738.26$ & 60,000 & 6004.57 & 5410.6 & 5296 & 3410.8 \\
32 days & $57,738.26$ & 64,000 & 4676.25 & 3897.5 & 3746.2 & 2114.3 \\
65 days & $55,934.21$ & 52,000 & $11,327.08$ & 12,994 & 12,487 & 10,459 \\
65 days & $55,934.21$ & 56,000 & 9593.51 & 10,596 & 10,746 & 8596.9 \\
65 days & $55,955.65$ & 60,000 & 8140.86 & 8871.5 & 8432.1 & 7037.7 \\
65 days & $55,955.65$ & 64,000 & 6910.42 & 6989.7 & 6829.5 & 5726 \\
\hline
\end{tabular}

Prices, the DEJD model European Call Prices, the SV model European Call Prices. Such comparison is to check the impact of each model and its efficiency regarding how it approaches the real market's prices. For each model, we run $M=20000$ simulations.

The prices of the DEJDSVJ model approach well the market's prices followed by the prices of the DEJD model for all three expiry times over different strikes. The results show that the SV model is not a good model for the BTC market options pricing.

An error measure method is used to check the goodness of fit. For that purpose, there are many methods as the Root Mean Square Error, the Average Absolute Error, the Average Percentage Error (APE) used by [10]. We use the APE because of its ability to not skew error rate around or equal to zero ([10]). The formula is:

$$
\operatorname{APE}=\frac{1}{N} \cdot \sum_{i=1}^{N} \frac{\left|\tilde{C}_{i}-C_{i}\right|}{\omega}
$$

where,

$$
\omega=\frac{1}{N} \sum_{i=1}^{N} C_{i}
$$

$N$ is the number of options used, $C_{i}$, the market price and $\tilde{C}_{i}$ the price for a given model.

Table 3 contains the Average Percentage Error for each model at a given time of maturity.

The APE for the DEJDSVJ model is smaller meaning that model outperforms the other models. Furthermore, the three graphs in Figure 5 show the prices of 
Table 3. APE for each model at 18, 32, 65 days before maturity.

\begin{tabular}{cccc}
\hline Model & APE for 18 days & APE for 32 days & APE for 65 days \\
\hline DEJDSVJ & $4.2 \%$ & $8.3 \%$ & $7.4 \%$ \\
DEJD & $4.3 \%$ & $8.9 \%$ & $9.6 \%$ \\
SV & $17.9 \%$ & $26.7 \%$ & $11 \%$ \\
\hline
\end{tabular}
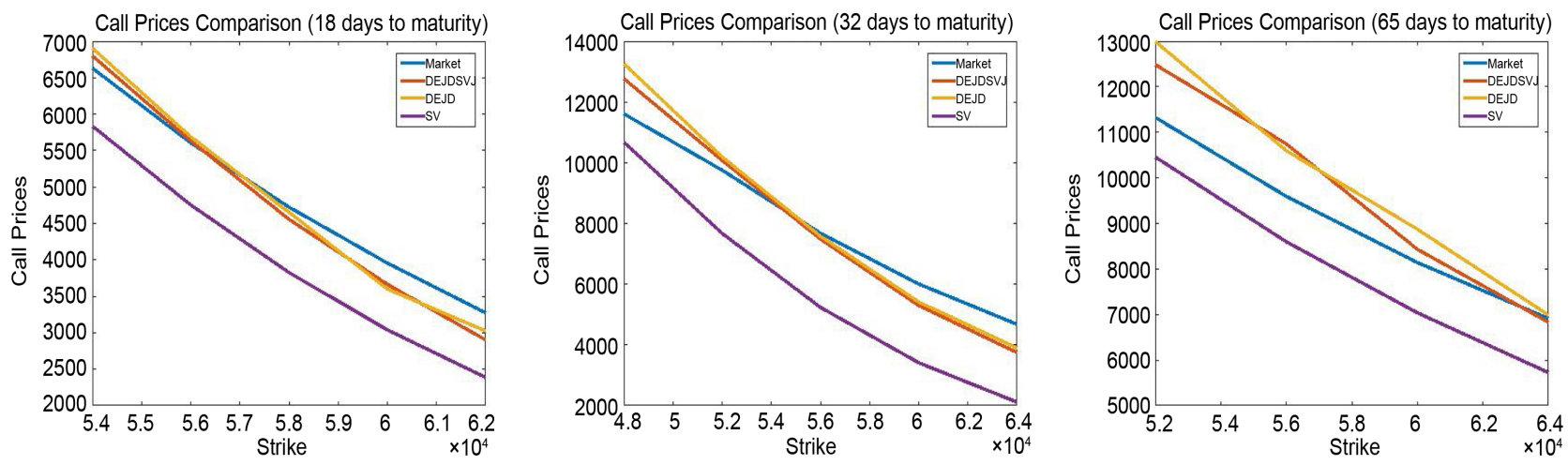

Figure 5. Call option prices of the BTC Options markets, the DEJD model, the DEJDSVJ model and the SV model.

the three models and the market prices against the strikes for each expiry. The curves of the DEJDSVJ's prices and the DEJD's prices are much closer to the market's prices. It confirms that the DEJDSVJ and DEJD models are better than the SV model when pricing options in BTC market.

We explain such performance of the DEJDSVJ model over other models by the incorporation of the jumps in both returns and volatility. That shows the recent importance of jumps when modelling financial assets.

\section{Implied Volatility Surface}

Implied Volatility captures the future expectations of a security's price. Forecasting the Implied Volatility is very important in trading and investments. It helps to buy cheap and to sell expensive in order to make a profit.

We analyze such volatility in this paper by drawing the Implied Volatility surface over Strikes and Maturity times. In Table 4, we have the European Call Options for different strikes and maturity times. We fix the current market price at $S=9000, M=20000$ M.C simulations and we use the parameters of the DEJD model to obtain the premiums. In Figure 6, we have the volatility surface of the option's premiums in Table 4. The surface shows two phenomenon:

- The long-dated options have higher Implied Volatility than the short-dated options;

- As the strike price increases, the Implied Volatility decreases.

As a result, it is profitable for traders and investors to buy strategies such that calls, puts with short expiry date (low Implied Volatility) and to sell strategies that have long maturity time. 
Table 4. Call option prices for the double exponential jump diffusion model with stochastic volatility jump (DEJDSVJ) model.

\begin{tabular}{rcccccc}
\hline Strike K & & & & & & \\
Maturity T & 1 & 7 & 30 & 60 & 90 & 180 \\
\hline 8000 & 1015.1 & 1160.5 & 1720.1 & 2304.9 & 2873.3 & 4703 \\
8100 & 910.3 & 1072.3 & 1635.3 & 2272.7 & 2846.5 & 4666.6 \\
8200 & 815.4 & 993.3 & 1576.6 & 2179.1 & 2734.1 & 4503.1 \\
8300 & 718.2 & 913.5 & 1502.3 & 2183 & 2725 & 4432.9 \\
8400 & 619.5 & 838.6 & 1448.4 & 2029.4 & 2649.4 & 4388.5 \\
8500 & 530.7 & 774.9 & 1378 & 2013 & 2613.6 & 4192.7 \\
8600 & 441.7 & 682 & 1321.7 & 1905.3 & 2583.2 & 4269.8 \\
8700 & 359.3 & 640.6 & 1235.7 & 1900.2 & 2485.2 & 4159.1 \\
8800 & 282.9 & 584.8 & 1193.7 & 1806.1 & 2435.8 & 4212.9 \\
8900 & 214.2 & 516.6 & 1140.1 & 1768.5 & 2340.1 & 4196 \\
9000 & 161.2 & 460.2 & 1071.7 & 1743.8 & 2268.2 & 4127.5 \\
9100 & 115.9 & 411.8 & 1038.1 & 1687.7 & 2272.2 & 3923.1 \\
9200 & 79.1 & 366.3 & 960.8 & 1645.9 & 2189.8 & 4013.6 \\
9300 & 53 & 311.2 & 954.7 & 1560.6 & 2117.5 & 3825.4 \\
9400 & 34.3 & 283.2 & 883.7 & 1489.4 & 2074.9 & 3783.3 \\
\hline
\end{tabular}

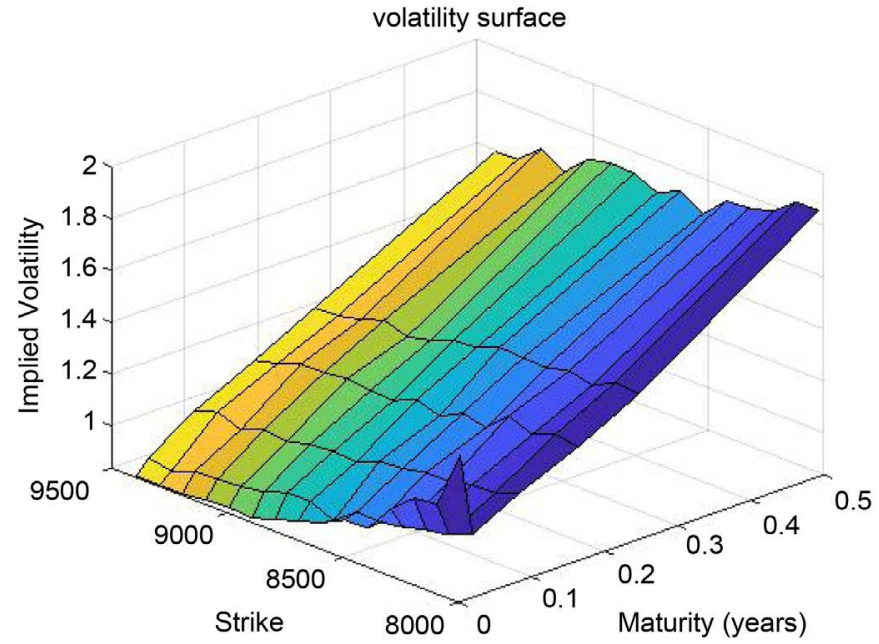

Figure 6. Implied volatility surface.

\section{Conclusions}

Recently, digital currencies gain a wide attention from economists, investors, traders and academicians, etc. Bitcoin, the first released cryptocurrency is the most popular because of its price's fluctuations (USD price) and the high level of volatility. Such interest challenges people to think about the future of Bitcoin and to improve more studies about it that will be helpful for traders and miners to avoid losses.

Following that wave, this paper extends the literature about jump-diffusion 
models for Bitcoin market by introducing a Double Exponential Jump-Diffusion model combined with a Stochastic Volatility process that incorporates asymmetric jumps. Theoretically, the choice of such model is supported by the important features captured by jumps in both returns and volatility as Bitcoin is too volatile. The importance of the double exponential distribution is its memoryless property and it allows to see easily the difference between the magnitudes (upward and downward) and intensities of jumps via the parameters $\eta_{1}, \eta_{2}, \eta_{1}^{\prime}, \eta_{2}^{\prime}, p, q$.

As pricing options can help for portfolios management, the mechanism of call options pricing using the underlying DEJDSVJ model shows much better performance than the DEJD model (no jumps in volatility) that outperforms the Stochastic Volatility where jumps are missing in both returns and volatility. Such outcomes also support the model.

A final conclusion is our results that meet the expectations.

The results presented in this paper concern only the Bitcoin data under Jump-Diffusion model in which the jumps are asymmetric Double exponentially distributed. However, for further works, one can consider the Pareto-Beta Jump-Diffusion that also allows for two approaches towards jumps' magnitudes in order to explore the Bitcoin market.

\section{Acknowledgements}

We acknowledge the African Union through the Pan African University, Institute of Basic Sciences, Technology and Innovation for its considerable support.

\section{Conflicts of Interest}

All authors have approved that there is not conflict of interest regarding the publication of this article.

\section{References}

[1] Nakamoto, S. (2008) Bitcoin: A Peer-to-Peer Electronic Cash System. White Paper.

[2] Brandvold, M., Molnár, P., Vagstad, K. and Valstad, O.C.A. (2015) Price Discovery on Bitcoin Exchanges. Journal of International Financial Markets, Institutions and Money, 36, 18-35. https://doi.org/10.1016/j.intfin.2015.02.010

[3] Dyhrberg, A.H. (2016) Bitcoin, Gold and the Dollar-A GARCH Volatility Analysis. Finance Research Letters, 16, 85-92. https://doi.org/10.1016/j.frl.2015.10.008

[4] Baur, D.G., Hong, K. and Lee, A.D. (2018) Bitcoin: Medium of Exchange or Speculative Assets? Journal of International Financial Markets, Institutions and Money, 54, 177-189. https://doi.org/10.1016/j.intfin.2017.12.004

[5] Black, F. and Scholes, M. (1973) The Pricing of Options and Corporate Liabilities. Journal of Political Economy, 81, 637-654. https://doi.org/10.1086/260062

[6] Kou, S.G. (2002) A Jump-Diffusion Model for Option Pricing. Management Science, 48, 1086-1101. https://doi.org/10.1287/mnsc.48.8.1086.166

[7] Hou, A.J., Wang, W.N., Chen, C.Y.H. and Hardle, W.K. (2020) Pricing Cryptocurrency Options. Journal of Financial Econometrics, 18, 250-279.

[8] Heston, S.L. (1993) A Closed-Form Solution for Options with Stochastic Volatility 
with Applications to Bond and Currency Options. The Review of Financial Studies, 6, 327-343. https://doi.org/10.1093/rfs/6.2.327

[9] Chen, R.D., Li, Z.X., Zeng, L.Y., Yu, L., Lin, Q. and Liu, J. (2017) Option Pricing under the Double Exponential Jump-Diffusion Model with Stochastic Volatility and Interest Rate. Journal of Management Science and Engineering, 2, 252-289. https://doi.org/10.3724/SP.J.1383.204012

[10] da Silva, F.C. (2018) Option Pricing under Jump-Diffusion Processes: Calibration to the Bitcoin Options Market. PhD Thesis.

[11] Pagnottoni, P. (2020) Neural Network Models for Bitcoin Option Pricing. Frontiers in Artificial Intelligence, 2, 5. https://doi.org/10.3389/frai.2019.00005

[12] Kjaerland, F., Khazal, A., Krogstad, E.A., et al. (2018) An Analysis of Bitcoin's Price Dynamics. Journal of Risk and Financial Management, 11, 63. https://doi.org/10.3390/jrfm11040063

[13] Nguyen, T., de Bodisco, C. and Thaver, R. (2018) Factors Affecting Bitcoin Price in the Cryptocurrency Market: An Empirical Study. International Journal of Business \& Economics Perspectives, 13, 106-125.

[14] Chen, C.Y.-H., Hardle, W.K., Hou, A.J. and Wang, W.N. (2018) Pricing Cryptocurrency Options: The Case of Crix and Bitcoin.

[15] Scaillet, O., Treccani, A. and Trevisan, C. (2020) High-Frequency Jump Analysis of the Bitcoin Market. Journal of Financial Econometrics, 18, 209-232.

[16] Mitra, S. (2009) Regime Switching Stochastic Volatility with Perturbation Based Option Pricing. https://doi.org/10.1504/IJFMD.2010.032470

[17] Tiwari, A.K., Kumar, S. and Pathak, R. (2019) Modelling the Dynamics of Bitcoin and Litecoin: Garch versus Stochastic Volatility Models. Applied Economics, 51, 4073-4082. https://doi.org/10.1080/00036846.2019.1588951

[18] Ramezani, C.A. and Zeng, Y. (2004) An Empirical Assessment of the Double Exponential Jump-Diffusion Process. https://doi.org/10.2139/ssrn.606101

[19] Tuzov, N. (2006) GMM Parameter Estimation for the Double Exponential Jump-Duffusion Process. Purdue University, West Lafayette.

[20] Cont, R. and Tankov, P. (2009) Constant Proportion Portfolio Insurance in the Presence of Jumps in Asset Prices. Mathematical Finance: An International Journal of Mathematics, Statistics and Financial Economics, 19, 379-401. https://doi.org/10.1111/j.1467-9965.2009.00377.x

[21] Jacquier, E., Polson, N.G. and Rossi, P.E. (2002) Bayesian Analysis of Stochastic Volatility Models. Journal of Business \& Economic Statistics, 20, 69-87. https://doi.org/10.1198/073500102753410408

[22] Eraker, B., Johannes, M. and Polson, N. (2003) The Impact of Jumps in Volatility and Returns. The Journal of Finance, 58, 1269-1300. https://doi.org/10.1111/1540-6261.00566

[23] Johannes, M. and Polson, N. (2009) Markov Chain Monte Carlo. In: Handbook of Financial Time Series, Springer, Berlin, 1001-1013. https://doi.org/10.1007/978-3-540-71297-8 43

[24] Asgharian, H. and Bengtsson, C. (2006) Jump Spillover in International Equity Markets. Journal of Financial Econometrics, 4, 167-203.

https://doi.org/10.1093/jifinec/nbj005 